\title{
Self-consistent DFT $+U$ method for real-space time-dependent density functional theory calculations
}

\author{
Nicolas Tancogne-Dejean,,$^{1,2, *}$ Micael J. T. Oliveira, ${ }^{1,2, \dagger}$ and Angel Rubio ${ }^{1,2,3, \dagger}$ \\ ${ }^{1}$ Max Planck Institute for the Structure and Dynamics of Matter, Luruper Chaussee 149, D-22761 Hamburg, Germany \\ ${ }^{2}$ European Theoretical Spectroscopy Facility (ETSF), Luruper Chaussee 149, 22761 Hamburg, Germany \\ ${ }^{3}$ Nano-Bio Spectroscopy Group, Universidad del País Vasco, 20018 San Sebastián, Spain
}

(Received 19 November 2017; published 22 December 2017)

\begin{abstract}
We implemented various DFT $+U$ schemes, including the Agapito, Curtarolo, and Buongiorno Nardelli functional (ACBNO) self-consistent density-functional version of the DFT $+U$ method [Phys. Rev. X 5, 011006 (2015)] within the massively parallel real-space time-dependent density functional theory (TDDFT) code OCTOPUS. We further extended the method to the case of the calculation of response functions with real-time $\mathrm{TDDFT}+U$ and to the description of noncollinear spin systems. The implementation is tested by investigating the ground-state and optical properties of various transition-metal oxides, bulk topological insulators, and molecules. Our results are found to be in good agreement with previously published results for both the electronic band structure and structural properties. The self-consistent calculated values of $U$ and $J$ are also in good agreement with the values commonly used in the literature. We found that the time-dependent extension of the self-consistent $\mathrm{DFT}+U$ method yields improved optical properties when compared to the empirical TDDFT $+U$ scheme. This work thus opens a different theoretical framework to address the nonequilibrium properties of correlated systems.
\end{abstract}

DOI: 10.1103/PhysRevB.96.245133

\section{INTRODUCTION}

It was recognized very early that the simplest local and semilocal approximations of density functional theory (DFT) fail to describe the electronic and structural properties of correlated materials, like transition-metal oxides [1,2]. Over the years, the DFT $+U$ method originally proposed by Anisimov, Lichtenstein, and coworkers [1,3-5] has become a wellestablished and successful way to improve the treatment of correlated solids in DFT. The physical motivation of the DFT $+U$ method, most often referred to as $\mathrm{LDA}+U$, stems from the overdelocalization of the electrons when treated within the local-density approximation (LDA) [6] or generalized gradient approximations (GGA) [7]. In particular, the localization of $3 d$ and $4 f$ electrons turns out to be extremely important for ground-state and excited-state properties of transition metals. In order to correct this overdelocalization, including an energy penalty, called $U$ in reference to the notation used in Hubbard Hamiltonians, for these orbitals was proposed [1-5]. The success of the DFT $+U$ method originates mainly from the simplicity of the method, its relatively low computational cost, and the fact that it can predict the proper magnetic ground state of Mott insulators [1]. However, the method has some intrinsic deficiencies, such as yielding infinite lifetimes for quasiparticles and opening the band gap by making a longrange order. Therefore, methods going beyond the DFT $+U$ method, that is, going beyond the mean-field level, such as the dynamical mean-field theory (DMFT) $[8,9]$ and, more recently, DFT+DMFT [10], have become the state-of-the-art methods to treat strongly correlated materials. These methods have been developed in depth in recent years, at the price of higher computational cost than DFT-type methods. As such, the DFT $+U$ approach still remains very attractive when it comes to the calculation of larger systems.

\footnotetext{
*nicolas.tancogne-dejean@mpsd.mpg.de

†micael.oliveira@mpsd.mpg.de

†angel.rubio@mpsd.mpg.de
}

In its original formulation, the DFT $+U$ method is not $a b$ initio, as no formal recipe was proposed to obtain the Hubbard $U$ and Hund $J$ parameters. Recently, various schemes have been proposed to obtain the value of the Hubbard $U$ $a b$ initio, instead of adjusting the values of $U$ and $J$ to spectroscopic data. Among them, one finds linear response calculations in supercells [11], constrained random-phase approximation [12-15], and direct approximations of the (screened) Hartree-Fock energy [16,17]. In this context, the work of Agapito et al. [16] is highly relevant as they introduced an efficient pseudohybrid functional, the Agapito, Curtarolo, and Buongiorno Nardelli functional (ACBNO), to obtain the $U$ and $J$ values $a b$ initio and self-consistently. This is achieved, in principle, with only a small increase in the computational cost when compared to standard LDA and GGA calculations. It was shown that the self-consistency in the calculation of the Hubbard $U$ can be crucial in the case of transition-metal complexes [18]. Furthermore, having a method that allows one to dynamically compute the value of the Hubbard $U$ is of fundamental relevance to describe correlated systems out of equilibrium.

This paper is organized as follows. First, we briefly present the usual DFT $+U$ method and the ACBN0 functional along with details of our implementation in the real-space code OCTOPUS [19-21]. Then, we present numerical results obtained with our implementation for the ground state of a bulk topological insulator in Sec. III, for the energy and forces of the isolated $\mathrm{O}_{2}$ molecule in Sec. IV, and for optical properties of two transition-metal oxides in Sec. V. Finally, we draw our conclusions in Sec. VI.

\section{DFT+ $U$ IN REAL SPACE}

In essence, the DFT $+U$ method replaces the DFT totalenergy functional $E_{\mathrm{DFT}}[n]$ with the $\mathrm{DFT}+U$ total-energy functional of the form

$$
\begin{aligned}
E_{\mathrm{DFT}+U}\left[n,\left\{n_{m m^{\prime}}^{I, \sigma}\right\}\right]= & E_{\mathrm{DFT}}[n]+E_{e e}\left[\left\{n_{m m^{\prime}}^{I, \sigma}\right\}\right] \\
& -E_{d c}\left[\left\{n_{m m^{\prime}}^{I, \sigma}\right\}\right],
\end{aligned}
$$


where $E_{e e}$ is the electron-electron interaction energy and $E_{d c}$ accounts for the double counting of the electron-electron interaction already present in $E_{\mathrm{DFT}}$. Although an exact form of the double-counting term was recently proposed in the context of DFT+DMFT [22], this double-counting term is not known in the general case, and several approximated forms have been proposed over the years.

The $E_{e e}$ and $E_{d c}$ energies depend on the density matrix of a localized orbital basis set $\left\{\phi_{I, m}\right\}$, which has localized orbitals attached to the atom $I$. In the following, we refer to the elements of the density matrix of the localized basis as occupation matrices, and we denote them as $\left\{n_{m m^{\prime}}^{I, \sigma}\right\}$. In the rotational-invariant form of $\mathrm{DFT}+U$ proposed by Dudarev et al. [23], one has

$$
\begin{aligned}
& E_{e e}\left[\left\{n_{m m^{\prime}}^{I, \sigma}\right\}\right]=\frac{U}{2} \sum_{m, m^{\prime}, \sigma} N_{m}^{\sigma} N_{m^{\prime}}^{-\sigma}+\frac{U-J}{2} \sum_{m \neq m^{\prime}, \sigma} N_{m}^{\sigma} N_{m^{\prime}}^{\sigma}, \\
& E_{d c}\left[\left\{n_{m m^{\prime}}^{I, \sigma}\right\}\right]=\frac{U}{2} N(N-1)-\frac{J}{2} N\left(\frac{N}{2}-1\right),
\end{aligned}
$$

where $N=N^{\uparrow}+N^{\downarrow}$ and $N^{\sigma}=\sum_{m} n_{m m}^{\sigma}$. Combining these two expressions, we obtain the $E_{U}$ energy to be added to the DFT total energy, which depends only on an effective Hubbard $U$ parameter $U^{\text {eff }}=U-J$,

$$
\begin{aligned}
E_{U}\left[\left\{n_{m m^{\prime}}^{I, \sigma}\right\}\right] & =E_{e e}\left[\left\{n_{m m^{\prime}}^{I, \sigma}\right\}\right]-E_{d c}\left[\left\{n_{m m^{\prime}}^{I, \sigma}\right\}\right] \\
& =\sum_{I, n, l} \frac{U_{I, n, l}^{\mathrm{eff}}}{2} \sum_{m, \sigma}\left(n_{m m}^{I, n, l, \sigma}-\sum_{m^{\prime}} n_{m m^{\prime}}^{I, n, l, \sigma} n_{m^{\prime} m}^{I, n, l, \sigma}\right),
\end{aligned}
$$

where $I$ is an atom index; $n, l$, and $m$ refer to the principal, azimuthal, and angular quantum numbers, respectively; and $\sigma$ is the spin index. In the case of a periodic system, the occupation matrices $n_{m m^{\prime}}^{I, n, l, \sigma}$ are given by

$$
n_{m m^{\prime}}^{I, n, l, \sigma}=\sum_{n} \sum_{\mathbf{k}}^{\mathrm{BZ}} w_{\mathbf{k}} f_{n \mathbf{k}}^{\sigma}\left\langle\psi_{n, \mathbf{k}}^{\sigma} \mid \phi_{I, n, l, m}\right\rangle\left\langle\phi_{I, n, l, m^{\prime}} \mid \psi_{n, \mathbf{k}}^{\sigma}\right\rangle,
$$

where $w_{\mathbf{k}}$ is the k-point weight and $f_{n \mathbf{k}}^{\sigma}$ is the occupation of the Bloch state $\left|\psi_{n, \mathbf{k}}^{\sigma}\right\rangle$. Here, $\left|\phi_{I, n, l, m}\right\rangle$ are the localized orbitals that form the basis used to describe electron localization.

From this energy, the corresponding set of generalized Kohn-Sham equations is obtained by minimizing Eq. (1) with respect to the wave functions for fixed occupations, which reads [24-26]

$$
\frac{\delta E_{\mathrm{DFT}+U}\left[n,\left\{n_{m m^{\prime}}^{I, \sigma}\right\}\right]}{\delta\left(\psi_{i}^{\sigma}\right)^{*}}-\frac{\delta \sum_{j} E_{j} f_{j}\left\langle\psi_{j} \mid \psi_{j}\right\rangle}{\delta\left(\psi_{i}^{\sigma}\right)^{*}}=0,
$$

where $i$ and $j$ refer to both band and $\mathbf{k}$-point indexes in the case of periodic systems. By comparing the resulting equations with the usual Kohn-Sham equations, one obtains the expression of the (nonlocal) potential that must be added to the DFT Hamiltonian. This potential reads

$$
V_{U}^{\sigma}\left|\psi_{n, \mathbf{k}}^{\sigma}\right\rangle=\sum_{I, n, l} \sum_{m, m^{\prime}} V_{m, m^{\prime}}^{I, n, l, \sigma} P_{m, m^{\prime}}^{I, n, l}\left|\psi_{n, \mathbf{k}}^{\sigma}\right\rangle
$$

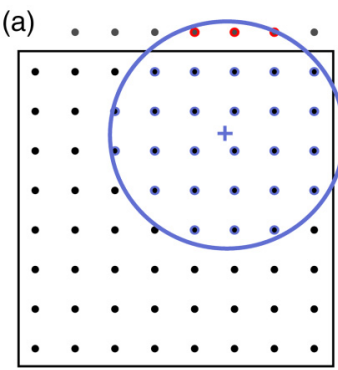

(b)

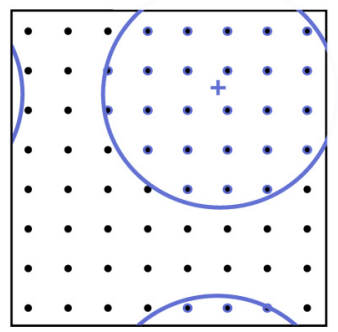

FIG. 1. (a) A localized orbital, represented on a subgrid (violet circle), overlaps with the border of the simulation box. Red points indicate grid points that belong to the subgrid of the localized orbital but not to the regular real-space grid. (b) The modified subgrid of the orbital, which is compatible with the periodicity of the solid and with the real-space grid. A phase shift is added to the points which are originally outside of the simulation box.

where we defined

$$
\begin{aligned}
V_{m, m^{\prime}}^{I, n, l, \sigma} & =U_{I, n, l}^{\mathrm{eff}}\left(\frac{1}{2} \delta_{m m^{\prime}}-n_{m m^{\prime}}^{I, n, l, \sigma}\right), \\
P_{m m^{\prime}}^{I, n, l} & =\left|\phi_{I, n, l, m}\right\rangle\left\langle\phi_{I, n, l, m^{\prime}}\right| .
\end{aligned}
$$

One important point of the implementation of the DFT $+U$ method lies in the choice and the representation of the localized basis. In the OCTOPUS code, which is a norm-conserving pseudopotential-based code, the natural choice is to use the pseudoatomic wave functions. The pseudo wave functions are usually decomposed into a radial part and an angular part given by the usual spherical harmonics but can easily be expressed on the real-space grid. Regarding this, it is worthwhile to note that some care is needed when treating periodic systems. Indeed, in bulk materials, the orbitals can spread over several unit cells. Therefore, one must at the same time describe an orbital bigger than the size of the simulation box and preserve periodicity for the main real-space grid. We solved this issue by representing the localized orbitals on spherical subgrids, centered around atoms, in a way very similar to the treatment of the Kleinman-Bylander projectors entering the expression of the nonlocal representation of the pseudopotentials. This is illustrated in Fig. 1. Finally, when computing projections of Bloch wave functions onto the localized atomic-orbital basis, a phase correction must be added to properly treat the periodicity when atomic orbitals cross the border of the simulation box.

Ultimately, the radii of the atomic spheres should be big enough to contain the entire localized orbitals. This can, however, correspond to a prohibitive number of real-space grid points, and in some cases, the radii of the atomic spheres must be reduced to obtain a good compromise between accuracy and computational efficiency. We implemented three methods to choose the radii of these atomic spheres. In the first method the atomic spheres are truncated at the radius of the nonlocal part of the pseudopotential, as proposed in Ref. [27]. The second method consists of truncating the radii to the smallest dimension of the unit cell. In the last method the radius of the spheres is chosen at the point where the radial component of the pseudo wave functions becomes smaller than some given threshold. 


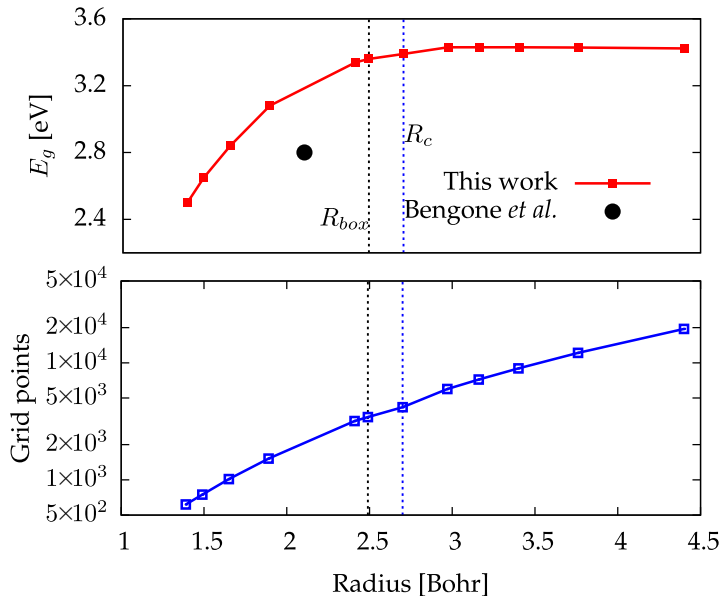

FIG. 2. Top: Convergence of the band gap of bulk NiO versus the truncation radius of the $d$ orbitals of nickel. We used $U_{\text {eff }}=U-J$ with $U=5.0 \mathrm{eV}$ and $J=0.95 \mathrm{eV}$, as chosen in Ref. [28]. The back dot corresponds to the value obtained in Ref. [28] for an atomic sphere radius of 2.1 bohrs. Bottom: Number of grid points covered by the $d$ orbitals of Ni. Two dashed vertical lines indicate, respectively, the cutoff radius $R_{c}$ of the nonlocal part of the pseudopotential used and the smallest dimension of the simulation box $R_{\mathrm{box}}$.

In order to illustrate the effect of such truncation, we studied the change in the band gap of bulk $\mathrm{NiO}$ in its antiferromagnetic phase with respect to the radius of the $3 d$ orbital of $\mathrm{Ni}$ for a fixed value of $U^{\text {eff }}$. We used a value of $4.1704 \AA$ for the lattice parameter of $\mathrm{NiO}$, with a real-space spacing of $\Delta r=0.3 \mathrm{bohr}$ and an $8 \times 8 \times 8 \mathbf{k}$-point grid to sample the Brillouin zone. We used the Perdew-Burke-Ernzerhof (PBE) functional [7] for the (semi)local DFT part. Our results, presented in Fig. 2, show that the band gap of $\mathrm{NiO}$ described using $\mathrm{DFT}+U$ strongly depends on the radius of the sphere used for the $3 d$ orbitals of the Ni atoms.

Interestingly, restricting the orbitals to a too small sphere results in an underestimation of the band gap, which, in turn, might be compensated by an overestimation of the value of the effective Hubbard $U$. As a comparison, we also report from the literature the band gap of $\mathrm{NiO}$ computed for the same value of $U^{\text {eff }}$ using the projector augmented-wave (PAW) method. [28] Most of the PAW and linear muffin-tin orbital (LMTO) implementations of the DFT $+U$ method $[5,24,28,29]$ are based on the so-called atomic-sphere approximation (ASA). In a pseudopotential-based code, such as the OCTOPUS code, this approximation is not necessary. However, in the spirit of ASA, one could decide to truncate the localized orbitals to the radius of the nonlocal part of the pseudopotential. Our results reveal that restricting the localized orbitals to the nonlocal part of the pseudopotential, as proposed in Ref. [27], can yield a good estimate of the band gap for a moderate number of grid points. However, it is worthwhile to note that the cutoff radius of a pseudopotential strongly depends on the method used to generate it. For instance, for nickel one can generate reasonable pseudopotentials whose cutoff radius varies from 1.5 to 2.5 bohrs. In the following, our results are always converged in total energy with respect to the size of the atomic spheres.

\section{III. $A B$ INITIO DFT $+U$ : THE ACBNO FUNCTIONAL}

In Ref. [16], an approximation to the electron interaction energy called the ACBNO functional was proposed, allowing for an efficient $a b$ initio evaluation of the DFT $+U$ energy and therefore of the DFT $+U$ Hamiltonian. In particular, only the computation of a reduced number of Coulomb integrals is needed to evaluate the effective Hubbard $U$. In the following, we always assume orthogonality of the localized basis set attached to each atom. This is obviously the case in our implementation, as the norm-conserving atomic pseudo wave functions are orthogonal by construction. Expressions corresponding to the general (nonorthogonal) case are already presented in Ref. [16] and are therefore not reported here. The electron interaction energy is given for the ACBN0 functional by [16]

$$
\begin{aligned}
E_{e e} & =\frac{1}{2} \sum_{\{m\}} \sum_{\alpha, \beta} \bar{P}_{m m^{\prime}}^{\alpha} \bar{P}_{m^{\prime \prime} m^{\prime \prime \prime}}^{\beta}\left(m m^{\prime} \mid m^{\prime \prime} m^{\prime \prime \prime}\right) \\
& -\frac{1}{2} \sum_{\{m\}} \sum_{\alpha} \bar{P}_{m m^{\prime}}^{\alpha} \bar{P}_{m^{\prime \prime} m^{\prime \prime \prime}}^{\alpha}\left(m m^{\prime \prime \prime} \mid m^{\prime \prime} m^{\prime}\right),
\end{aligned}
$$

corrected here for a small typographical mistake [the minus sign between the two terms in Eq. (6)] in the original paper. In Eq. (6), the renormalized occupation matrices $\bar{P}_{m m^{\prime}}^{I, n, l, \sigma}$ and the renormalized occupations $\bar{N}_{\psi_{n \mathbf{k}}}^{I, n, l, \sigma}$ are, respectively, given by

$$
\begin{gathered}
\bar{P}_{m m^{\prime}}^{I, n, l, \sigma}=\sum_{n \mathbf{k}} w_{\mathbf{k}} f_{n \mathbf{k}} \bar{N}_{\psi_{n \mathbf{k}}}^{I, n, l, \sigma}\left\langle\psi_{n \mathbf{k}}^{\sigma} \mid \phi_{I, n, l, m}\right\rangle\left\langle\phi_{I, n, l, m^{\prime}} \mid \psi_{n \mathbf{k}}^{\sigma}\right\rangle, \\
\bar{N}_{\psi_{n \mathbf{k}}}^{I, n, l, \sigma}=\sum_{\{I\}} \sum_{m}\left\langle\psi_{n \mathbf{k}}^{\sigma} \mid \phi_{I, n, l, m}\right\rangle\left\langle\phi_{I, n, l, m} \mid \psi_{n \mathbf{k}}^{\sigma}\right\rangle
\end{gathered}
$$

where the sums in Eq. (8) run over all orbitals of the system having the quantum numbers $n$ and $l$ and being attached to atoms of the same type as atom $I$, as this quantity is similar to the Mulliken charge of atom $I$ [16].

From Eq. (6), the effective Hubbard $U$ is given by $U^{\text {eff }}=$ $\bar{U}-\bar{J}$, where [16]

$$
\begin{gathered}
\bar{U}=\frac{\sum_{\{m\}} \sum_{\alpha \beta} \bar{P}_{m m^{\prime}}^{\alpha} \bar{P}_{m^{\prime} m^{\prime \prime}}^{\beta}\left(m m^{\prime} \mid m^{\prime \prime} m^{\prime \prime \prime}\right)}{\sum_{m \neq m^{\prime}} \sum_{\alpha} N_{m}^{\alpha} N_{m^{\prime}}^{\alpha}+\sum_{\{m\}} \sum_{\alpha} N_{m}^{\alpha} N_{m^{\prime}}^{-\alpha}}, \\
\bar{J}=\frac{\sum_{\{m\}} \sum_{\alpha} \bar{P}_{m m^{\prime}}^{\alpha} \bar{P}_{m^{\prime} m^{\prime \prime}}^{\alpha}\left(m m^{\prime \prime \prime} \mid m^{\prime \prime} m^{\prime}\right)}{\sum_{m \neq m^{\prime}} \sum_{\alpha} N_{m}^{\alpha} N_{m^{\prime}}^{\alpha}},
\end{gathered}
$$

with $N_{m}^{\alpha}=n_{m m}^{I, n, l, \alpha}$. Here, we omitted the atom and quantum number subscripts for conciseness. Altogether, these equations define the ACBN0 functional. It is important to note that $0 \leqslant$ $\bar{N}_{\psi_{n \mathrm{k}}}^{I, n, l, \sigma} \leqslant 1$. In the limit of $\bar{N}_{\psi_{n \mathbf{k}}}^{I, n, l, \sigma}=0$, we recover the DFT energy, whereas for $\bar{N}_{\psi_{n \mathrm{k}}}^{I, n, l, \sigma}=1$ we have the Hartree-Fock energy. However, the energy given by the ACBNO function lies in between, which is usually a prerequisite for obtaining reliable electronic gaps [30].

The potential corresponding to the ACBNO functional should, in principle, be obtained by solving Eq. (4) using Eqs. (2), (9), and (10). This leads to an orbital-dependent potential, which must be obtained using the optimized effective potential (OEP) method [31-33]. Here, however, we decided to implement the functional as defined in the original ACBN0 paper, where the usual DFT $+U$ potential of Eq. (5) is used. 
TABLE I. Values of $U_{\text {eff }}$ (in $\mathrm{eV}$ ) for various transition-metal oxides, obtained from our implementation, compared to the result of the original ACBN0 paper [16].

\begin{tabular}{lccccc}
\hline \hline \multirow{2}{*}{ Material } & \multicolumn{2}{c}{ TM 3d } & & \multicolumn{2}{c}{ Oxygen 2p } \\
\cline { 2 - 3 } \cline { 5 - 6 } & This work & Ref. [16] & & This work & Ref. [16] \\
\hline $\mathrm{MnO}$ & 4.68 & 4.67 & & 5.18 & 2.68 \\
$\mathrm{NiO}$ & 6.93 & 7.63 & & 2.87 & 3.0 \\
$\mathrm{TiO}_{2}$ & 0.96 & 0.15 & & 10.18 & 7.34 \\
$\mathrm{ZnO}$ & 13.3 & 12.8 & & 5.95 & 5.29 \\
\hline \hline
\end{tabular}

In Table I, we report the values of the effective $U$ obtained with our implementation, compared with the results of the original ACBN0 paper [16]. We also report in Table II the band gaps of transition-metal oxides obtained by our implementation compared to the results obtained in the original ACBN0 paper [16] and to experimental results. We employed a real-space grid spacing of $0.25 \mathrm{bohr}$ and an $8 \times 8 \times 8 \mathbf{k}$ point grid to sample the Brillouin zone. We considered $\mathrm{TiO}_{2}$ in its rutile phase with experimental lattice constants $a=b=4.594 \AA, c=2.959 \AA$, and $\mu=0.305$ [34] and $\mathrm{ZnO}$ in its wurtzite phase with lattice constants $a=b=3.1995 \AA$, $c=5.1330 \AA$, and $\mu=0.3816[16,34]$. As for $\mathrm{MnO}$, we considered it in its ideal rocksalt structure, as already done above for $\mathrm{NiO}$, with type-II antiferromagnetic spin ordering and with a lattice constant of $a=4.4315 \AA$. In all the cases, we considered localization on both the $3 d$ orbitals of the transition-metal atoms and the $2 p$ orbitals of the oxygen atoms.

Overall, our results are found to be in good agreement with the ones reported in Ref. [16]. However, it is worth noting that one of the difficulties when implementing the ACBNO functional lies in the calculation of the Coulomb integrals. In Ref. [16], the authors make used of a $3 \mathrm{G}$ Gaussian basis set to help calculate the Coulomb integrals, as it is not possible to compute them directly in reciprocal space. A possible origin for discrepancies can therefore be attributed to different treatment of the localized orbitals, which are not approximated here by a sum of three Gaussians, as we express them directly on the real-space grid. We also compare the band structures of $\mathrm{ZnO}$ and $\mathrm{TiO}_{2}$ calculated from our implementation to the results of Ref. [16] in Figs. 3 and 4, showing good agreement.

The ACBN0 functional has been shown to improve structural and magnetic properties [35,36]. We also found that the ACBN0 functional yields improved electronic structures

TABLE II. Values of the direct band gap (in eV) for various transition-metal oxides, obtained from our implementation, compared to the result of the original ACBN0 paper and to experimental values (see Ref. [16] and references therein).

\begin{tabular}{lccc}
\hline \hline Material & This work & Ref. [16] & Expt. \\
\hline $\mathrm{MnO}$ & 2.65 & 2.83 & $4.1 ; 3.9 \pm 0.4 ; 3.8-4.2 ; 3.6-3.8$ \\
$\mathrm{NiO}$ & 4.14 & 4.29 & $4.0 ; 4.3 ; 3.7 ; 3.7 ; 3.87$ \\
$\mathrm{TiO}_{2}$ & 3.21 & 2.83 & $3.3 \pm 0.5 ; 3.03$ \\
$\mathrm{ZnO}$ & 3.04 & 2.91 & $3.3 ; 3.44 ; 3.44$ \\
\hline \hline
\end{tabular}

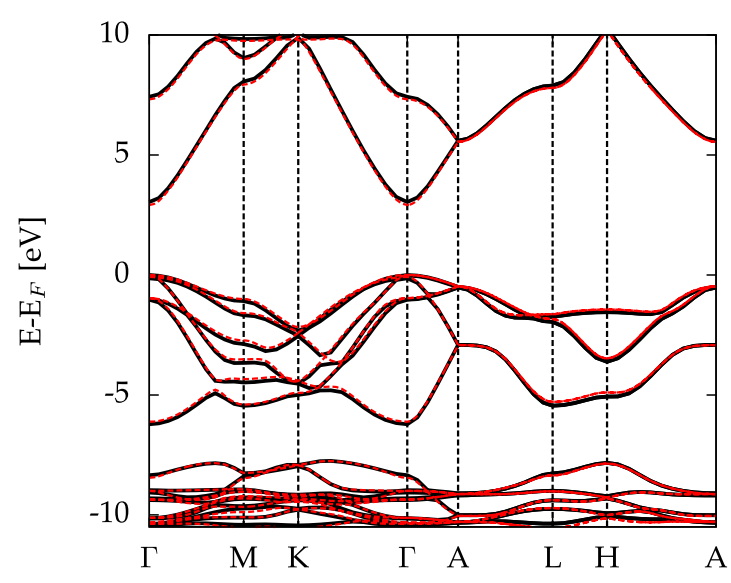

FIG. 3. Band structure of wurtzite zinc oxide, calculated from our implementation (solid black lines) compared to the result of the original ACBN0 paper (dashed red lines) [16].

for various types of materials, such as three-dimensional topological insulators. First, we report in Fig. 5 the band structure of $\mathrm{Sb}_{2} \mathrm{Te}_{3}$, which is a bulk topological insulator, computed without spin-orbit coupling (SOC). In this case we employed Hartwigsen-Goedecker-Hutter pseudopotentials [37], a real-space grid spacing of $0.35 \mathrm{bohr}$, and an $8 \times 8 \times$ 8 k-point grid to sample the Brillouin zone and used the experimental constant for the lattice parameters and internal constants [38]. Similar to previously reported results [39], our results show that the LDA band structure exhibits an avoided crossing at the Fermi energy close to the $\Gamma$ point (see the right panel of Fig. 5). By including an effective Hubbard $U$ for the $5 p$ orbitals of Te in our description, computed from the ACBN0 functional, we open the band gap of this material. We obtained values of $U_{\text {eff }}=2.95 \mathrm{eV}$ and $U_{\text {eff }}=2.80 \mathrm{eV}$ for the Te atom at Wyckoff position $3 a$ and for the two other equivalent Te atoms (Wyckoff position $6 c$ ), respectively. The calculated band gap is $0.23 \mathrm{eV}$, in reasonable agreement with the reported full GW band gap of $0.10 \mathrm{eV}$ [39]. It is worth noting here that a one-shot GW calculation leads, for this material, to an unphysical band dispersion close to the Fermi energy, and off-diagonal matrix elements must be included

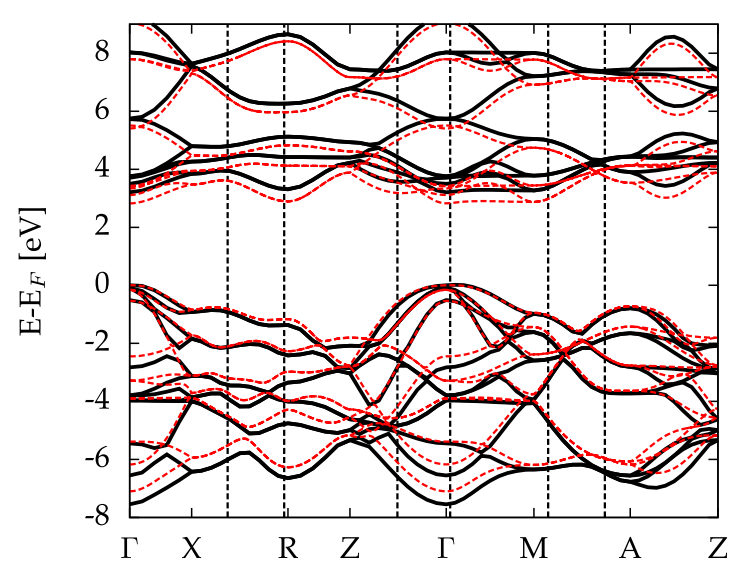

FIG. 4. Same as Fig. 3, but for $\mathrm{TiO}_{2}$ in its rutile phase. 

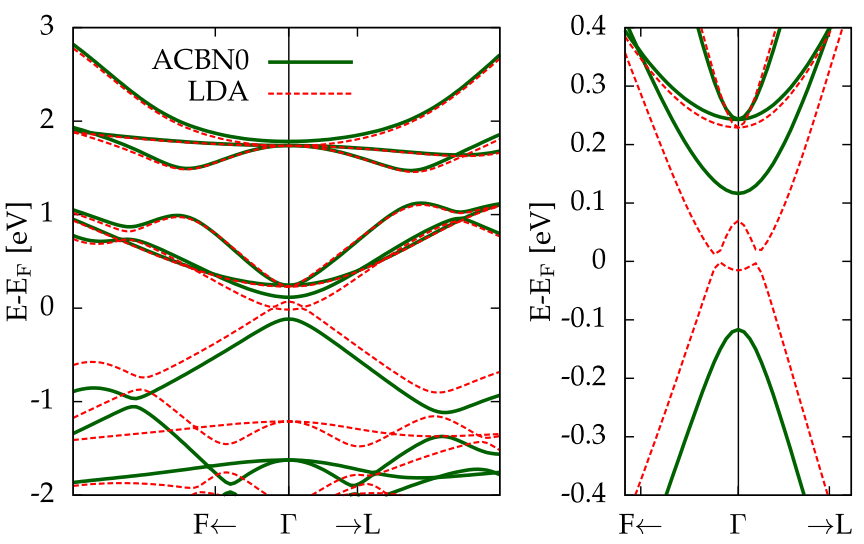

FIG. 5. Left: Band structure of bulk topological insulation $\mathrm{Sb}_{2} \mathrm{Te}_{3}$, calculated using the LDA functional (dashed red lines) and using the ACBN0 functional (solid green lines). Right: Details of the band-structure around the $\Gamma$ point.

in the self-energy to correctly dehybridize the bands (see Ref. [39] for a detailed discussion).

In the previous results the $\mathrm{SOC}$ was neglected. However, the topological nature of $\mathrm{Sb}_{2} \mathrm{Te}_{3}$ and related materials originates from the spin-orbit interaction. We have therefore extended the ACBN0 functional to the case of noncollinear spin in order to be able to describe such materials. The main formulas of DFT $+U$ in the case of noncollinear spin are presented in Appendix A, and the extension of the ACBN0 functional with noncollinear spin is presented in Appendix B. In Fig. 6 we compare the band structure of $\mathrm{Sb}_{2} \mathrm{Te}_{3}$ computed with $\mathrm{SOC}$ using the LDA and ACBN0 functionals. Again, we find that our results are in good agreement with the GW results of Ref. [39] for the same material and that the unphysical band dispersion around the $\Gamma$ point for LDA disappears using the ACBN0 functional. We obtained values of $U_{\text {eff }}=2.89 \mathrm{eV}$ and $U_{\text {eff }}=2.78 \mathrm{eV}$ for the Te atom at Wyckoff position $3 a$ and for the two other equivalent Te atoms (Wyckoff position $6 c$ ), respectively. These values are very similar to the values obtained without SOC. The corresponding band gap is $0.21 \mathrm{eV}$, in excellent agreement with the reported full GW with a SOC

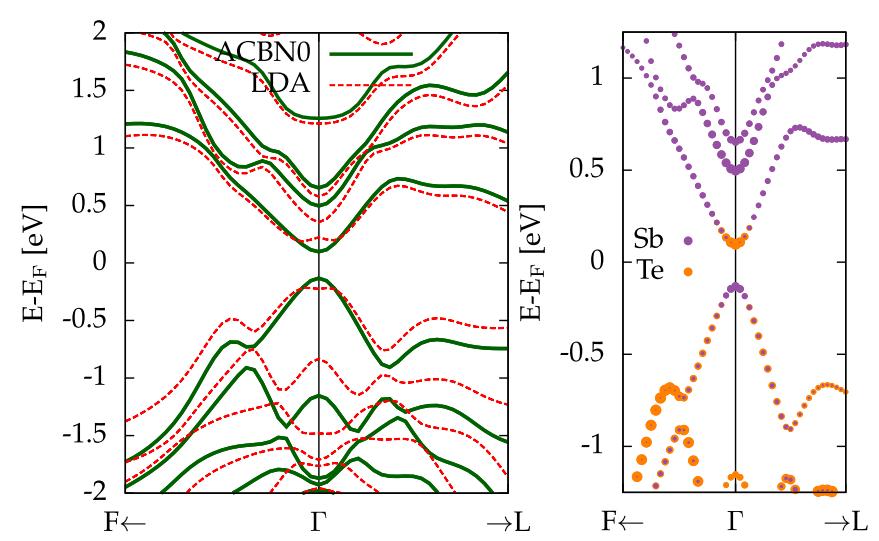

FIG. 6. Left: Same as the left panel of Fig. 5, but now including full spin-orbit coupling (see the text for details of dealing with spinors in the ACBN0 functional). Right: The $p$-orbital character of the band structure around the $\Gamma$ point. band gap of $0.21 \mathrm{eV}$ of Ref. [39]. As shown in the right panel of Fig. 6, a band inversion around the $\Gamma$ point is found with the ACBN0 functional, as expected from the topological nature of $\mathrm{Sb}_{2} \mathrm{Te}_{3}$ and in agreement with previous LDA and GW results [39]. Our results indicate that the ACBN0 functional seems to be a good alternative to the computationally expensive full-GW calculation for describing bulk topological insulators such as $\mathrm{Sb}_{2} \mathrm{Te}_{3}$.

\section{FORCES AND ISOLATED SYSTEMS}

In many relevant physical situations, the knowledge of forces is important. Due to the modification of the total energy of the system done in Eq. (1), the contribution to the forces, sometimes referred to as Hubbard forces, must be computed. The contribution to the forces acting on atom $\alpha$ in direction $i$ coming from the Hubbard energy of atom $I$ and the quantum numbers $n$ and $l$ is defined by

$$
\begin{aligned}
F_{\alpha, i}^{U} & =-\frac{\partial E_{U}}{\partial R_{\alpha, i}}=-\sum_{I, m, m^{\prime}, \sigma} \frac{\partial E_{U}}{\partial n_{m m^{\prime}}^{I, n, l, \sigma}} \frac{\partial n_{m m^{\prime}}^{I, n, l, \sigma}}{\partial R_{\alpha, i}} \\
& =-\frac{U}{2} \sum_{I, m, m^{\prime}, \sigma}\left(\delta_{m m^{\prime}}-2 n_{m m^{\prime}}^{I, n, l, \sigma}\right) \frac{\partial n_{m m^{\prime}}^{I, n, \sigma}}{\partial R_{\alpha, i}} .
\end{aligned}
$$

From the definition of the occupation matrices for periodic systems, one finds that

$$
\begin{aligned}
\frac{\partial n_{m m^{\prime}}^{I, \sigma}}{\partial R_{\alpha, i}}= & \sum_{\mathbf{k}, v} w_{\mathbf{k}} f_{\mathbf{k}, v}\left[\frac{\partial\left\langle\phi_{m, \mathbf{k}}^{I, n, l} \mid \psi_{\mathbf{k}, v}^{\sigma}\right\rangle}{\partial R_{\alpha, i}}\left\langle\psi_{\mathbf{k}, v}^{\sigma} \mid \phi_{m, \mathbf{k}}^{I, n, l}\right\rangle\right. \\
& \left.+\left\langle\phi_{m, \mathbf{k}}^{I} \mid \psi_{\mathbf{k}, v}^{\sigma}\right\rangle \frac{\partial\left\langle\psi_{\mathbf{k}, v}^{\sigma} \mid \phi_{m, \mathbf{k}}^{I}\right\rangle}{\partial R_{\alpha, i}}\right] .
\end{aligned}
$$

Instead of using this expression directly, we compute the forces from the derivatives of the orbitals in order to reduce the so called egg-box effect [21]. After some algebra, one can show that

$$
\begin{aligned}
\frac{\partial n_{m m^{\prime}}^{I, n, l, \sigma}}{\partial R_{\alpha, i}}= & \delta_{\alpha, I} \sum_{\mathbf{k}, v} w_{\mathbf{k}} f_{\mathbf{k}, v}\left[\left\langle\phi_{m^{\prime}, \mathbf{k}}^{I, n, l} \mid \frac{\partial \psi_{\mathbf{k}, v}^{\sigma}}{\partial r_{i}}\right\rangle\left\langle\psi_{\mathbf{k}, v}^{\sigma} \mid \phi_{m, \mathbf{k}}^{I, n, l}\right\rangle\right. \\
& \left.+\left\langle\phi_{m^{\prime}, \mathbf{k}}^{I, n, l} \mid \psi_{\mathbf{k}, v}^{\sigma}\right\rangle\left\langle\frac{\partial \psi_{\mathbf{k}, v}^{\sigma}}{\partial r_{i}} \mid \phi_{m, \mathbf{k}}^{I, n, l}\right\rangle\right] .
\end{aligned}
$$

We illustrate how our implementation works with a simple, but relevant and widely studied, case: the variation of the binding energy and the forces of the $\mathrm{O}_{2}$ dimer in its triplet ground state ${ }^{3} \Sigma_{g}^{-}$. We employed a real-space grid spacing of $0.2 \mathrm{bohr}$, and we fixed the occupations to be the ones of the triplet ${ }^{3} \Sigma_{g}^{-}$state. We found (see Fig. 7) that the binding energy and the forces are both consistent in predicting a smaller equilibrium distance when the Hubbard $U$ is added, thus showing the reliability of our implementation. Interestingly, the self-consistent Hubbard $U$ obtained from the ACBN0 functional predicts an equilibrium distance closer to the experimental distance of $1.208 \AA$ [40] than those using the PBE functional or the $\mathrm{PBE}+U$ with a fixed empirical $U$ of $4 \mathrm{eV}$, as summarized in Table III. 

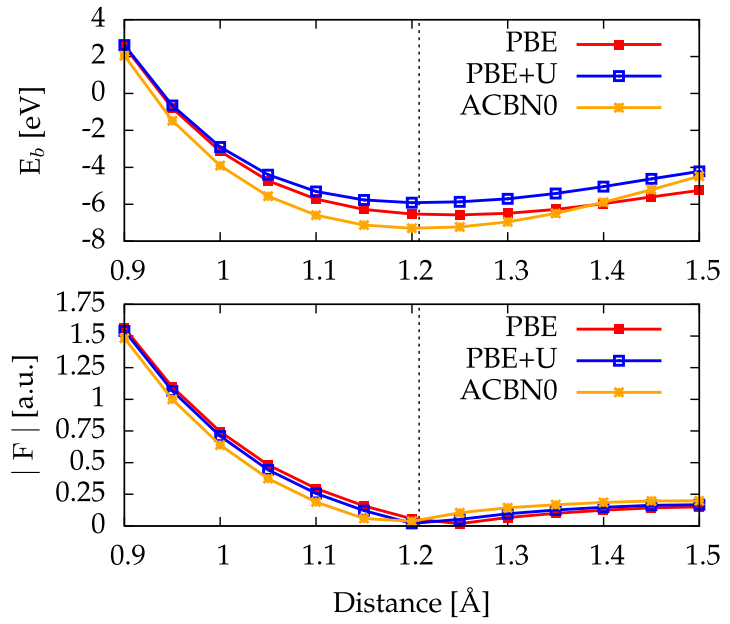

FIG. 7. Top: Binding energy versus atomic distance for the $\mathrm{O}_{2}$ molecule, obtained from $\mathrm{PBE}$ (blue curve), $\mathrm{PBE}+U$ with $U=4 \mathrm{eV}$ (red curve), and using the ACBN0 functional (orange curve). Bottom: Corresponding absolute value of the atomic force. The vertical line indicates the experimental equilibrium distance of $1.208 \AA$ [40].

\section{REAL-TIME TIME-DEPENDENT DFT $+U$ CALCULATIONS: OPTICAL RESPONSE FUNCTIONS}

Excited-state properties cannot be obtained from groundstate DFT. Therefore, we extended our DFT $+U$ implementation to also support real-time time-dependent $\mathrm{DFT}+U$ $(\mathrm{TDDFT}+U)$. Following the usual approach used when extending time-independent functionals to the time-dependent case, we make the adiabatic approximation, which in our case means that

$$
\begin{aligned}
V_{U}^{\sigma}\left(\mathbf{r}, \mathbf{r}^{\prime}, t\right) & =\left\langle\mathbf{r}\left|\hat{V}_{U}^{\sigma}(t)\right| \mathbf{r}^{\prime}\right\rangle \\
& =V_{U}^{\sigma}\left[n(t),\left\{n_{m m^{\prime}}^{\sigma}\right\}(t), U^{\mathrm{eff}}(t)\right]\left(\mathbf{r}, \mathbf{r}^{\prime}\right)
\end{aligned}
$$

This corresponds to evaluating the potential defined by Eq. (5) from the density, occupation matrices, and effective $U$ computed from the time-dependent wave functions.

Real-time TDDFT $+U$ is an alternative to the more commonly used linear response TDDFT $+U$, where optical properties are computed from perturbation theory based on the energies and wave functions obtained from ground $\mathrm{DFT}+U$ calculations [28]. In OCTOPUS, we implemented the real-time TDDFT $+U$ method for both the usual $\mathrm{DFT}+U$ scheme (presented in Sec. II) and the ab initio scheme based on the ACBN0 functional (see Sec. III).

Since the DFT $+U$ potential of Eq. (14) is a nonlocal operator, some care is required when extending the DFT $+U$ method to the time-dependent case. In order to preserve gauge

TABLE III. Calculated values of the equilibrium bond length of $\mathrm{O}_{2}$ and vibration frequencies obtained for the different methods compared to the corresponding experimental value.

\begin{tabular}{lcccc}
\hline \hline Method & PBE & PBE+U & ACBN0 & Expt. \\
\hline Bond length $(\AA)$ & 1.245 & 1.218 & 1.210 & 1.208 \\
Vibration frequency $\left(\mathrm{cm}^{-1}\right)$ & 1552 & 1506 & 1773 & 1580 \\
\hline \hline
\end{tabular}

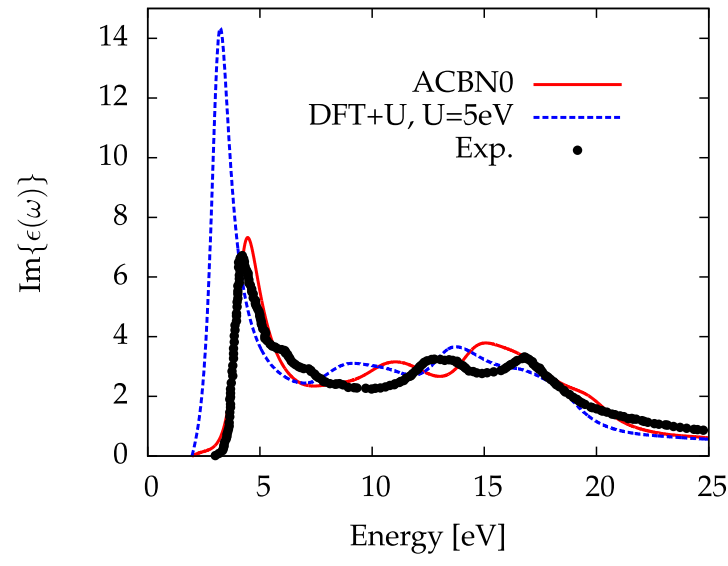

FIG. 8. Calculated optical absorption spectra of bulk NiO obtained using the ACBN0 functional (solid red line) and the usual DFT $+U$ scheme with $U_{\text {eff }}=5 \mathrm{eV}$ (dashed blue line). The experimental data are taken from Ref. [41]. A Gaussian broadening of $\eta=0.5 \mathrm{eV}$ is added to reproduce the experimental broadening.

invariance, we replace Eq. (14) by

$$
\hat{V}_{U}^{\sigma, \mathbf{A}}(t)\left|\psi_{n, \mathbf{k}}^{\sigma}(t)\right\rangle=e^{-i \mathbf{A}(t) \hat{\mathbf{r}}} \hat{V}_{U}^{\sigma}(t) e^{i \mathbf{A}(t) \hat{\mathbf{r}}}\left|\psi_{n, \mathbf{k}}^{\sigma}(t)\right\rangle,
$$

where $\mathbf{A}(t)$ denotes the vector potential perturbing the electronic system. Moreover, the electronic current is modified such that

$$
\begin{array}{r}
\mathbf{j}_{\mathrm{DFT}+U}(\mathbf{r}, t)=\mathbf{j}_{\mathrm{DFT}}(\mathbf{r}, t) \\
-\frac{1}{2} \sum_{n} \sum_{\mathbf{k}}^{\mathrm{BZ}} w_{\mathbf{k}} f_{n, \mathbf{k}}\left\langle\psi_{n, \mathbf{k}}\left|\left[\hat{\mathbf{r}}, \hat{V}_{U}^{\sigma, \mathbf{A}}(t)\right]\right| \psi_{n, \mathbf{k}}\right\rangle+\text { c.c. },
\end{array}
$$

where c.c. denotes the complex conjugate.

This allows us, for instance, to study linear and nonlinear optical properties of transition-metal oxides without relying on perturbation theory. To illustrate this, we computed the absorption spectrum of bulk $\mathrm{NiO}$ in its antiferromagnetic phase. We used a time step of 0.01 a.u. and propagated the timedependent Kohn-Sham equations, after a sudden perturbation of the system along the [100] direction, up to 200 a.u. in order to get a converged spectra. Our results, presented in Fig. 8, show the absorption spectra of $\mathrm{NiO}$ obtained using the ACBN0 functional (red solid line) and using the usual DFT $+U$ scheme with a fixed $U$ of $5 \mathrm{eV}$ (blue dashed line). We considered localization for both the $3 d$ orbitals of the transition-metal atoms and the $2 p$ orbitals of the $\mathrm{O}$ atoms. We also report in Fig. 9 the optical spectrum of $\mathrm{MnO}$ in its antiferromagnetic phase.

The optical spectra obtained from the ACBN0 functional yields results in surprisingly good agreement with the experimental spectrum, much better in both pole structure and oscillator strength than the LDA ones. This indicates that it is possible to get at the same time good ground-state properties, such as the band gap, and good optical spectra using the $a b$ initio $\mathrm{DFT}+U$ method. We found that the excitonic effects are not captured in the absorption spectra of $\mathrm{TiO}_{2}$ and $\mathrm{ZnO}$ (not shown). Adding excitonic contributions to the spectra would be the next step to consider (as already done for normal semiconductors and insulators using the GW+BSE method [45]) and might be achieved by combining the present 


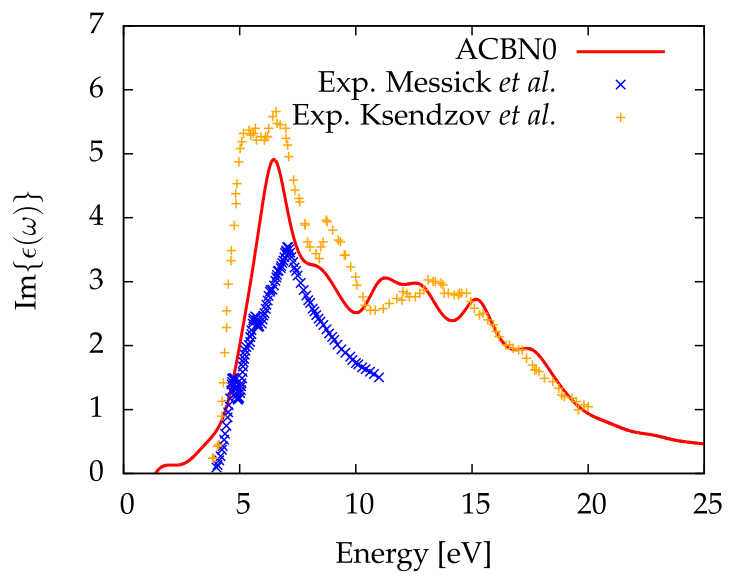

FIG. 9. Calculated optical absorption spectra of bulk $\mathrm{MnO}$ obtained using the ACBN0 functional (red line). The experimental data are taken from Messick et al. [42] and Ksendzov et al. [43,44]. A Gaussian broadening of $\eta=0.4 \mathrm{eV}$ is added to reproduce the experimental broadening.

work with recent developments in describing exciton dynamics using hybrid functionals. See, for instance, Ref. [46].

\section{CONCLUSION}

We reported an efficient implementation of the DFT $+U$ method and its extension to the time-dependent case in the real-space TDDFT code OCTOPUS. In the case of ground-state calculations, we showed that our implementation yields results similar to the ones previously reported in the literature. Moreover, our real-time TDDFT $+U$ implementation is capable of producing good absorption spectra of transition-metal oxides, based on the ACBNO functional, maintaining the relatively low cost of the TDDFT $+U$ approach while being fully ab initio.

\section{ACKNOWLEDGMENTS}

We acknowledge financial support from the European Research Council (Grant No. ERC-2015-AdG-694097), Grupos Consolidados UPV/EHU (IT578-13), and the European Union's H2020 program under Grant Agreement No. 676580 (NOMAD). N.T.-D. would like to acknowledge T. Brumme for interesting and fruitful discussions.

\section{APPENDIX A: DFT $+U$ WITH NONCOLLINEAR SPIN}

Starting from the Hartree-Fock energy of the localized basis for noncollinear spin, we can define $U_{I, n, l}$ and $J_{I, n, l}[47]$ and obtain

$$
\begin{aligned}
E_{e e}\left[\left\{n_{m m^{\prime}}^{I, m, l, \sigma \sigma^{\prime}}\right\}\right] & \\
= & \frac{U_{I, n, l}}{2} \sum_{\sigma} \sum_{m m^{\prime}} n_{m m}^{\sigma \sigma} n_{m^{\prime} m^{\prime}}^{-\sigma-\sigma}-\frac{U_{I, n, l}}{2} \sum_{\sigma} \sum_{m} n_{m m}^{\sigma-\sigma} n_{m m}^{-\sigma \sigma} \\
& -\frac{J_{I, n, l}}{2} \sum_{\sigma} \sum_{m \neq m^{\prime}} n_{m m}^{\sigma-\sigma} n_{m^{\prime} m^{\prime}}^{-\sigma \sigma} \\
& +\frac{U_{I, n, l}-J_{I, n, l}}{2} \sum_{\sigma} \sum_{m \neq m^{\prime}} n_{m m}^{\sigma \sigma} n_{m^{\prime} m^{\prime}}^{\sigma \sigma},
\end{aligned}
$$

where we assumed for simplicity that the occupation matrices are diagonal. These occupation matrices are matrices in spin space and read

$$
n_{m m^{\prime}}^{I, m, l, \sigma \sigma^{\prime}}=\sum_{n} \sum_{\mathbf{k}}^{\mathrm{BZ}} w_{\mathbf{k}} f_{n \mathbf{k}}\left\langle\psi_{n, \mathbf{k}}^{\sigma} \mid \phi_{I, n, l, m}^{\sigma}\right\rangle\left\langle\phi_{I, n, l, m^{\prime}}^{\sigma^{\prime}} \mid \psi_{n, \mathbf{k}}^{\sigma^{\prime}}\right\rangle,
$$

where $f_{n \mathbf{k}}$ is the occupation of the spinor state $\left|\psi_{n \mathbf{k}}\right\rangle$. Here, we consider the most general case, in which the localized basis is described by spinors, but this is not mandatory.

In order to get the DFT $+U$ energy, we need to remove the double-counting part. For this, we use the expression proposed in Ref. [48] for the fully localized limit,

$$
\begin{aligned}
E_{\mathrm{DC}}= & \frac{U_{I, n, l}}{2} N(N-1)-\frac{J_{I, n, l}}{2} N\left(\frac{N}{2}-1\right) \\
& -\frac{J_{I, n, l}}{4} \mathbf{m} \cdot \mathbf{m},
\end{aligned}
$$

where $N=\operatorname{Tr}_{l s}\left\{n_{m m^{\prime}}^{\sigma \sigma^{\prime}}\right\}$ is the number of electrons in the localized orbitals, i.e., $N=\sum_{\sigma} \sum_{m} n_{m m}^{\sigma \sigma}$, and $\mathbf{m}$ is the magnetization of the localized subspace. In turn, $\mathbf{m}$ is defined as

$$
\mathbf{m}=\operatorname{Tr}_{s}\{\sigma \cdot \rho\}, \quad \rho^{\sigma \sigma^{\prime}}=\operatorname{Tr}_{l}\left[n_{m m^{\prime}}^{\sigma \sigma^{\prime}}\right],
$$

where $\operatorname{Tr}_{l}$ is the trace over the orbitals, $\sigma_{i}$ are the Pauli matrices, and $\operatorname{Tr}_{s}$ is the trace over spins.

Putting everything together, one obtains that the rotationally invariant form corresponding to Eq. (2) for the noncollinear spins is

$$
E_{U}=\frac{U_{I, n, l}-J_{I, n, l}}{2}\left[\sum_{\sigma} \sum_{m} n_{m m}^{\sigma \sigma}-\sum_{m m^{\prime}} \sum_{\sigma \sigma^{\prime}} n_{m m^{\prime}}^{\sigma \sigma^{\prime}}{ }_{m^{\prime} m}^{\sigma^{\prime} \sigma}\right] .
$$

\section{APPENDIX B: ACBNO FUNCTIONAL WITH NONCOLLINEAR SPIN}

In order to derive the expressions needed to compute the ACBN0 functional for noncollinear spin, we first consider the Hartree-Fock energy corresponding to the $\{m\}$ subspace,

$$
\begin{aligned}
E_{e e}\left[\left\{n_{m m^{\prime}}^{I, m, l, \sigma \sigma^{\prime}}\right\}\right]= & \frac{1}{2} \sum_{\sigma \sigma^{\prime}} \sum_{\{m\}}\left[n_{m m^{\prime}}^{\sigma \sigma} n_{m^{\prime \prime} m^{\prime \prime \prime}}^{\sigma^{\prime}{ }^{\prime}}\left(m m^{\prime} \sigma \mid m^{\prime \prime} m^{\prime \prime \prime} \sigma^{\prime}\right)\right. \\
& \left.-n_{m m^{\prime}}^{\sigma^{\prime} \sigma} n_{m^{\prime \prime} m^{\prime \prime \prime}}^{\sigma \sigma^{\prime}}\left(m m^{\prime \prime \prime} \sigma \mid m^{\prime \prime} m^{\prime} \sigma^{\prime}\right)\right], \quad \text { (B1) }
\end{aligned}
$$

where the Coulomb integrals are denoted using the notation

$$
\begin{aligned}
& \left(m m^{\prime} \sigma \mid m^{\prime \prime} m^{\prime \prime \prime} \sigma^{\prime}\right) \\
& \quad=\left\langle m \sigma, m^{\prime \prime} \sigma^{\prime}|V| m^{\prime} \sigma, m^{\prime \prime \prime} \sigma^{\prime}\right\rangle \\
& \quad=\int d^{3} \mathbf{r}_{\mathbf{1}} \int d^{3} \mathbf{r}_{\mathbf{2}} \frac{\phi_{m}^{\sigma *}\left(\mathbf{r}_{\mathbf{1}}\right) \phi_{m^{\prime}}^{\sigma}\left(\mathbf{r}_{\mathbf{1}}\right) \phi_{m^{\prime \prime}}^{\sigma^{\prime} *}\left(\mathbf{r}_{2}\right) \phi_{m^{\prime \prime \prime}}^{\sigma^{\prime}}\left(\mathbf{r}_{\mathbf{2}}\right)}{\left|\mathbf{r}_{\mathbf{1}}-\mathbf{r}_{\mathbf{2}}\right|} .
\end{aligned}
$$

Following the idea of Agapito et al. [16], we introduce the "renormalized" occupation number $\bar{N}_{\Psi_{i}^{I, n},}^{I,}$ [see Eq. (8)]. We then define the renormalized density matrix as

$$
\bar{P}_{m m^{\prime}}^{I, n, l, \sigma \sigma^{\prime}}=\sum_{n \mathbf{k}} \bar{N}_{\Psi_{n \mathbf{k}}}^{I, n, l} w_{\mathbf{k}} f_{n \mathbf{k}}\left\langle\psi_{n \mathbf{k}}^{\sigma} \mid \phi_{m}^{\sigma}\right\rangle\left\langle\phi_{m^{\prime}}^{\sigma^{\prime}} \mid \psi_{n \mathbf{k}}^{\sigma^{\prime}}\right\rangle,
$$


with

$$
\bar{N}_{\psi_{n \mathbf{k}}}^{I, n, l}=\sum_{\{I\}} \sum_{m}\left\langle\psi_{n \mathbf{k}} \mid \phi_{I, n, l, m}\right\rangle\left\langle\phi_{I, n, l, m} \mid \psi_{n \mathbf{k}}\right\rangle .
$$

This definition reduces to the usual expression for the diagonal terms (in spin space) but also includes the screening (from the renormalization) for the off-diagonal terms.

Next, we replace $n_{m m^{\prime}}^{\sigma \sigma^{\prime}}$ by $\bar{P}_{m m^{\prime}}^{\sigma \sigma^{\prime}}$ in the previous expression and define $\bar{U}$ and $\bar{J}$ by comparing Eq. (B1) with Eq. (A1).

We obtain

$$
\begin{gathered}
\bar{U}=\frac{\sum_{\sigma \sigma^{\prime}} \sum_{\{m\}} \bar{P}_{m m^{\prime}}^{\sigma \sigma} \bar{P}_{m^{\prime \prime} m^{\prime \prime \prime}}^{\sigma^{\prime \prime} \sigma^{\prime}}\left(m m^{\prime} \sigma \mid m^{\prime \prime} m^{\prime \prime \prime} \sigma^{\prime}\right)}{\sum_{\sigma} \sum_{m m^{\prime}} N_{m}^{\sigma \sigma} N_{m^{\prime}}^{-\sigma-\sigma}+\sum_{\sigma} \sum_{m \neq m^{\prime}} N_{m}^{\sigma \sigma} N_{m^{\prime}}^{\sigma \sigma}-\sum_{\sigma} \sum_{m} N_{m}^{\sigma-\sigma} N_{m}^{-\sigma \sigma}}, \\
\bar{J}=\frac{\sum_{\sigma \sigma^{\prime}} \sum_{\{m\}} \bar{P}_{m m^{\prime}}^{\sigma \sigma^{\prime}} \bar{P}_{m^{\prime \prime} m^{\prime \prime \prime}}^{\sigma^{\prime} \sigma}\left(m m^{\prime \prime \prime} \sigma \mid m^{\prime \prime} m^{\prime} \sigma^{\prime}\right)}{\sum_{\sigma} \sum_{m \neq m^{\prime}}\left[N_{m}^{\sigma \sigma} N_{m^{\prime}}^{\sigma \sigma}+N_{m}^{\sigma-\sigma} N_{m^{\prime}}^{-\sigma \sigma}\right]},
\end{gathered}
$$

with $N_{m}^{\alpha \beta}=n_{m m}^{I, n, l, \alpha, \beta}$.

[1] V. I. Anisimov, F. Aryasetiawan, and A. Lichtenstein, J. Phys. Condens. Matter 9, 767 (1997).

[2] B. Himmetoglu, A. Floris, S. Gironcoli, and M. Cococcioni, Int. J. Quantum Chem. 114, 14 (2014).

[3] V. I. Anisimov, J. Zaanen, and O. K. Andersen, Phys. Rev. B 44, 943 (1991).

[4] V. I. Anisimov, I. V. Solovyev, M. A. Korotin, M. T. Czyżyk, and G. A. Sawatzky, Phys. Rev. B 48, 16929 (1993).

[5] A. I. Liechtenstein, V. I. Anisimov, and J. Zaanen, Phys. Rev. B 52, R5467(R) (1995).

[6] J. P. Perdew and Y. Wang, Phys. Rev. B 45, 13244 (1992).

[7] J. P. Perdew, K. Burke, and M. Ernzerhof, Phys. Rev. Lett. 77, 3865 (1996).

[8] W. Metzner and D. Vollhardt, Phys. Rev. Lett. 62, 324 (1989).

[9] A. Georges and G. Kotliar, Phys. Rev. B 45, 6479 (1992).

[10] G. Kotliar, S. Y. Savrasov, K. Haule, V. S. Oudovenko, O. Parcollet, and C. A. Marianetti, Rev. Mod. Phys. 78, 865 (2006).

[11] M. Cococcioni and S. de Gironcoli, Phys. Rev. B 71, 035105 (2005).

[12] T. Miyake and F. Aryasetiawan, Phys. Rev. B 77, 085122 (2008).

[13] M. Aichhorn, L. Pourovskii, V. Vildosola, M. Ferrero, O. Parcollet, T. Miyake, A. Georges, and S. Biermann, Phys. Rev. B 80, 085101 (2009).

[14] T. Miyake, F. Aryasetiawan, and M. Imada, Phys. Rev. B 80, 155134 (2009).

[15] M. Aichhorn, S. Biermann, T. Miyake, A. Georges, and M. Imada, Phys. Rev. B 82, 064504 (2010).

[16] L. A. Agapito, S. Curtarolo, and M. Buongiorno Nardelli, Phys. Rev. X 5, 011006 (2015).

[17] N. J. Mosey and E. A. Carter, Phys. Rev. B 76, 155123 (2007).

[18] H. J. Kulik, M. Cococcioni, D. A. Scherlis, and N. Marzari, Phys. Rev. Lett. 97, 103001 (2006).

[19] M. A. Marques, A. Castro, G. F. Bertsch, and A. Rubio, Comput. Phys. Commun. 151, 60 (2003).

[20] A. Castro, H. Appel, M. Oliveira, C. A. Rozzi, X. Andrade, F. Lorenzen, M. A. L. Marques, E. K. U. Gross, and A. Rubio, Phys. Status Solidi B 243, 2465 (2006).

[21] X. Andrade, D. Strubbe, U. De Giovannini, A. H. Larsen, M. J. T. Oliveira, J. Alberdi-Rodriguez, A. Varas, I. Theophilou, N. Helbig, M. J. Verstraete, L. Stella, F. Nogueira, A. Aspuru-
Guzik, A. Castro, M. A. L. Marques, and A. Rubio, Phys. Chem. Chem. Phys. 17, 31371 (2015).

[22] K. Haule, Phys. Rev. Lett. 115, 196403 (2015).

[23] S. L. Dudarev, G. A. Botton, S. Y. Savrasov, C. J. Humphreys, and A. P. Sutton, Phys. Rev. B 57, 1505 (1998).

[24] A. B. Shick, A. I. Liechtenstein, and W. E. Pickett, Phys. Rev. B 60, 10763 (1999).

[25] J. P. Perdew and A. Zunger, Phys. Rev. B 23, 5048 (1981).

[26] J. F. Janak, Phys. Rev. B 18, 7165 (1978).

[27] G. Sclauzero and A. Dal Corso, Phys. Rev. B 87, 085108 (2013).

[28] O. Bengone, M. Alouani, P. Blöchl, and J. Hugel, Phys. Rev. B 62, 16392 (2000).

[29] B. Amadon, F. Jollet, and M. Torrent, Phys. Rev. B 77, 155104 (2008).

[30] P. Mori-Sánchez, A. J. Cohen, and W. Yang, Phys. Rev. Lett. 100, 146401 (2008).

[31] R. T. Sharp and G. K. Horton, Phys. Rev. 90, 317 (1953).

[32] J. D. Talman and W. F. Shadwick, Phys. Rev. A 14, 36 (1976).

[33] S. Kümmel and J. P. Perdew, Phys. Rev. Lett. 90, 043004 (2003).

[34] S. C. Abrahams and J. L. Bernstein, J. Chem. Phys. 55, 3206 (1971).

[35] P. Gopal, R. D. Gennaro, M. S. dos Santos Gusmao, R. A. R. A. Orabi, H. Wang, S. Curtarolo, M. Fornari, and M. B. Nardelli, J. Phys. Condens. Matter 29, 444003 (2017).

[36] S. Lee, H. Wang, P. Gopal, J. Shin, H. M. I. Jaim, X. Zhang, S.-Y. Jeong, D. Usanmaz, S. Curtarolo, M. Fornari, M. Buongiorno Nardelli, and I. Takeuchi, Chem. Mater. 29, 9378 (2017).

[37] C. Hartwigsen, S. Goedecker, and J. Hutter, Phys. Rev. B 58, 3641 (1998).

[38] R. Wyckoff, Crystal Structures, 2nd ed. (Interscience, New York, 1963).

[39] I. Aguilera, C. Friedrich, G. Bihlmayer, and S. Blügel, Phys. Rev. B 88, 045206 (2013).

[40] K.-P. Huber, Molecular Spectra and Molecular Structure: IV. Constants of Diatomic Molecules (Springer, New York, 2013).

[41] R. J. Powell and W. E. Spicer, Phys. Rev. B 2, 2182 (1970).

[42] L. Messick, W. C. Walker, and R. Glosser, Phys. Rev. B 6, 3941 (1972). 
[43] Y. M. Ksendzov, I. L. Koroboa, K. K. Sidorin, and G. P. Startsev, Fiz. Tverd. Tela (Leningrad) 18, 173 (1976).

[44] C. Rödl and F. Bechstedt, Phys. Rev. B 86, 235122 (2012).

[45] G. Onida, L. Reining, and A. Rubio, Rev. Mod. Phys. 74, 601 (2002).
[46] S. Refaely-Abramson, M. Jain, S. Sharifzadeh, J. B. Neaton, and L. Kronik, Phys. Rev. B 92, 081204 (2015).

[47] B.-C. Shih, Y. Zhang, W. Zhang, and P. Zhang, Phys. Rev. B 85, 045132 (2012).

[48] F. Bultmark, F. Cricchio, O. Grånäs, and L. Nordström, Phys. Rev. B 80, 035121 (2009). 